\title{
LA POSTURA HOLANDESA FRENTE AL CONTROL DE LA FISURACIÓN
}

\section{(THE DUTCH ATTITUDE VERSUS CRACK CONTROL)}

\author{
Jan Stasse, Ingeniero \\ Consejero del ADVIESBUREAU ir. J.B. HAGEMAN c.i.B.V.- Rijewijk \\ HOLANDA
}

Fecha de recepción: 24-V-92

\begin{abstract}
RESUMEN
La gran tradición existente en Holanda, en la construcción de la Arquitectura con originales fábricas de albañilería, se mantiene en la actualidad buscando mejoras en los estándares de calidad.

Con el fin de poder garantizar las pólizas de seguro sobre la construcción, se ha creado la necesidad de desarrollar la investigación que permita evitar, en el tiempo, la aparición de fisuras en las fábricas.
\end{abstract}

El artículo expone las causas más comunes de la fisuración de las fábricas y la manera de controlarlas mediante la adecuada disposición de las armaduras.

\section{SUMMARY}

In the Netherlands, the long tradition which exists in building with original bricklaying masonries is still kept at present by seeking for improvements in the quality standards.

In order to be able to guarantee the insurance policies in construction, a need has been created to develop the research which would allow to avoid induced cracks in masonries.

This article explains the most common causes of masonry cracking and the way to control it through the adecuate layout of the reinforcements.

\section{INTRODUCCIÓN}

La albañilería era bien conocida en la antigüedad. La mayoría de las veces el mortero aplicado era de calidad pobre o incluso no se aplicaba.

Desde el primer momento se vio la necesidad de abrir huecos para puertas y ventanas en los edificios y viviendas, para poder acceder a iluminar el interior y hacerlo habitable.

Al principio, el dintel tenía grandes dimensiones como el ejemplo de la Puerta de los Leones, en Micenas-Creta (Foto 1).

Al cabo de algún tiempo los dinteles se sustituyeron por otros más sencillos de dimensiones considerables.
La utilización de otros materiales para dinteles, como la madera, también tuvieron una aplicación muy remota.

Así, pues, es evidente que la humanidad fue consciente de que el material de fábrica sobre un hueco no podía sostenerse por sí mismo y que debía de sujetarse por debajo, o bien colgarse desde arriba.

Esta segunda opción obligaba a considerar al mismo tiempo un vínculo de atado en la parte inferior de la fábrica (Fig. 1).

Pronto se supo que la densidad del material de fábrica, que constituye el arco, influiría directamente en la necesidad de adoptar un procedimiento para soportar los empujes horizontales que se pudieran generar. 


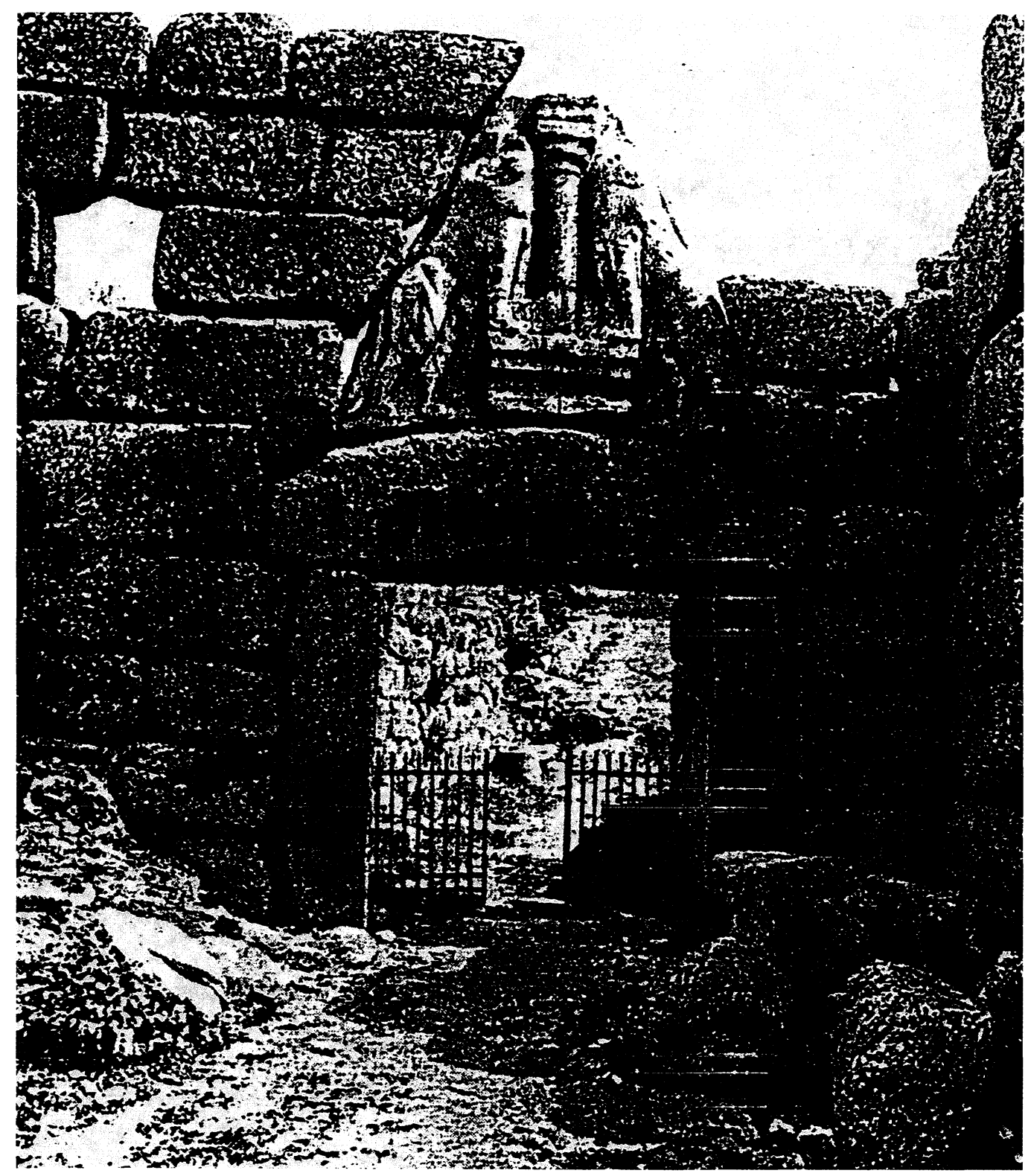

Foto 1.-Puerta de los Leones en Micenas.

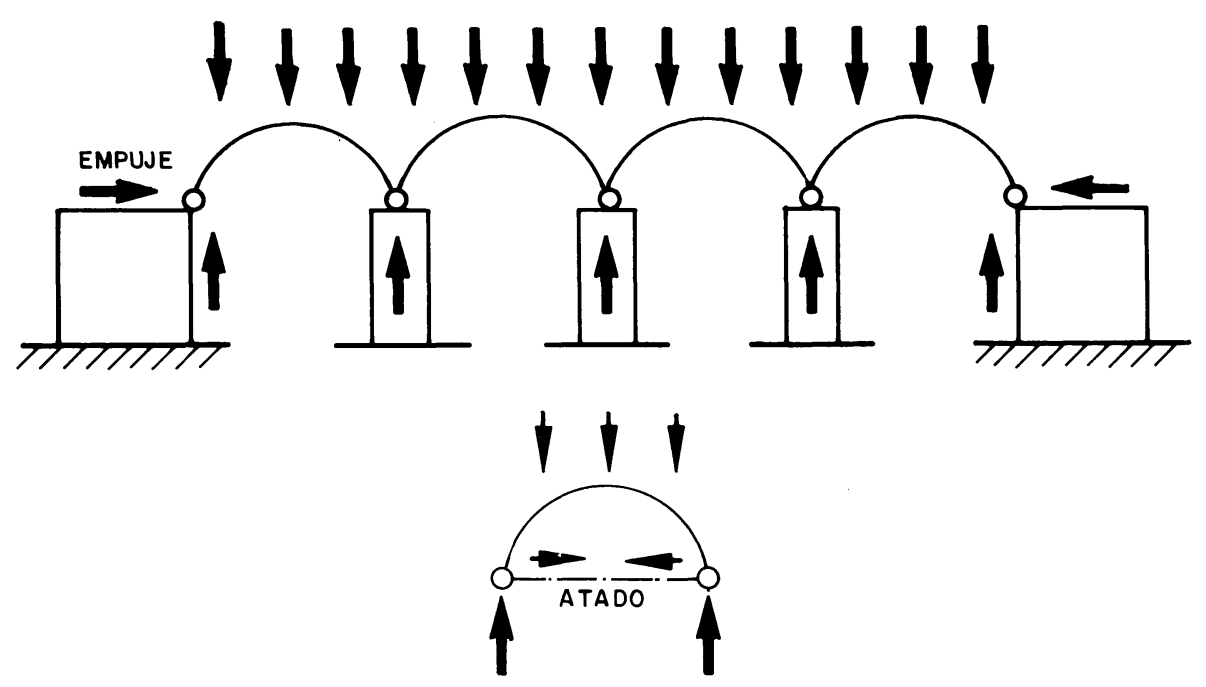

Fig. 1.-Absorción del empuje del arco mediante el atado del tirante, o colocación de estribos. 
Apenas es necesario decir que los casos mencionados previamente fueron, y aún lo son, aplicaciones mundiales en estructuras, y que Holanda no es ninguna excepción a este respecto. Sin embargo, la obra de fábrica holandesa es específica debida a algunos aspectos:

- En primer lugar, la piedra natural para material de construcción es difícil de encontrar en Holanda y, por tanto, se usa ladrillo de arcilla natural. Era el único producto existente hasta años después de la segunda guerra mundial.

Las dimensiones son bastantes pequeñas: hasta $215 \mathrm{~mm}$ de longitud, $103 \mathrm{~mm}$ de anchura y $52 \mathrm{de}$ altura.

En 1950, aproximadamente, se introdujo el ladrillo sílico-calcáreo, y algún tiempo después el ladrillo de hormigón, en las mismas dimensiones que el ladrillo de arcilla y al principio aplicados como un producto para particiones dentro de los edificios.

El ladrillo externo sílico-calcáreo se desarrolló más tarde, dando nuevas posibilidades a la arquitectura moderna. Todos estos ladrillos o bloques son piezas macizas. La aplicación de piezas huecas con cavidades en dirección horizontal o vertical, de modo que se pueda introducir armadura, no es un método corriente en Holanda.

- Un segundo aspecto de la práctica holandesa en albañilería era la demanda de diseñar estructuras de dimensiones limitadas, debido a las circunstancias, bastante desfavorables, para las cimentaciones.

Estratos de suelo con una capacidad portante más bien alta a nivel de superficie sólo se pueden encontrar en las regiones del Este del país, lo que ocasiona la necesidad de estructuras relativamente ligeras sobre pilotes en las regiones donde las capas sólidas están en niveles más bajos, hasta $25 \mathrm{~m}$ por debajo de la superficie.

- Un tercer aspecto es que, hasta la segunda guerra mundial, aproximadamente, las paredes de albañilería eran sólidas, con una anchura de unos $200 \mathrm{~mm}$.

La aplicación de muros dobles es ahora práctica habitual y ofrece la ventaja importante de colocar aislamiento térmico en la cavidad entre la pared interna y la externa.
La pared externa tiene por carga sólo su propio peso, pero debe resistir también fluctuaciones térmicas, tema importante para el diseño de estructuras de albañilería. Las fuerzas del viento son transportadas desde la pared externa a la pared interna por las llaves de acoplamiento (al menos $4 \mathrm{p} / \mathrm{m}^{2}$ ) entre ambas paredes, $\mathrm{y}$ después son transportadas a paredes transversales y suelos.

\section{APLICACIÓN DE LA FÁBRICA ARMADA EN HOLANDA}

En la construcción holandesa, el diseño de albañilería con aplicación de refuerzos está limitada al producto Murfor de BEKAERT.

En la secuencia de importancia respecto a las posibilidades de aplicación, el primer puesto es ocupado por:

1. El armado de atado o tirante en dinteles, en la hoja externa de las paredes dobles.

Los puestos siguientes son:

2. Armado en paredes interiores y exteriores no portantes de carga.

3. Armado en paredes de almacenamiento agrícola cargadas horizontalmente, tales como silos de patatas y bodegas o sótanos.

4. Armado de atado de hojas entre paredes internas y externas en muros dobles.

Desde luego, hay otras posibilidades de aplicación, pero la frecuencia de esas aplicaciones no es realmente significativa en la construcción holandesa.

Si consideramos con detalle la aplicación más importante, en la fábrica armada para dinteles se puede observar que su aplicación está impedida por la preferencia de muchos arquitectos holandeses de hacer juntas de dilatación verticales en una esquina, o incluso en ambas esquinas del hueco (Fotos 2 y 3 ).

De este modo la aplicación de la armadura debe formar un puente por encima de la junta, y el asentamiento de la junta debe ser contrarrestado.

La razón de este método de subdividir la pared viene de la experiencia de los arquitectos e ingenieros holandeses que detectan fisuras en esta zona, donde es habitual que surjan en sentido vertical. 

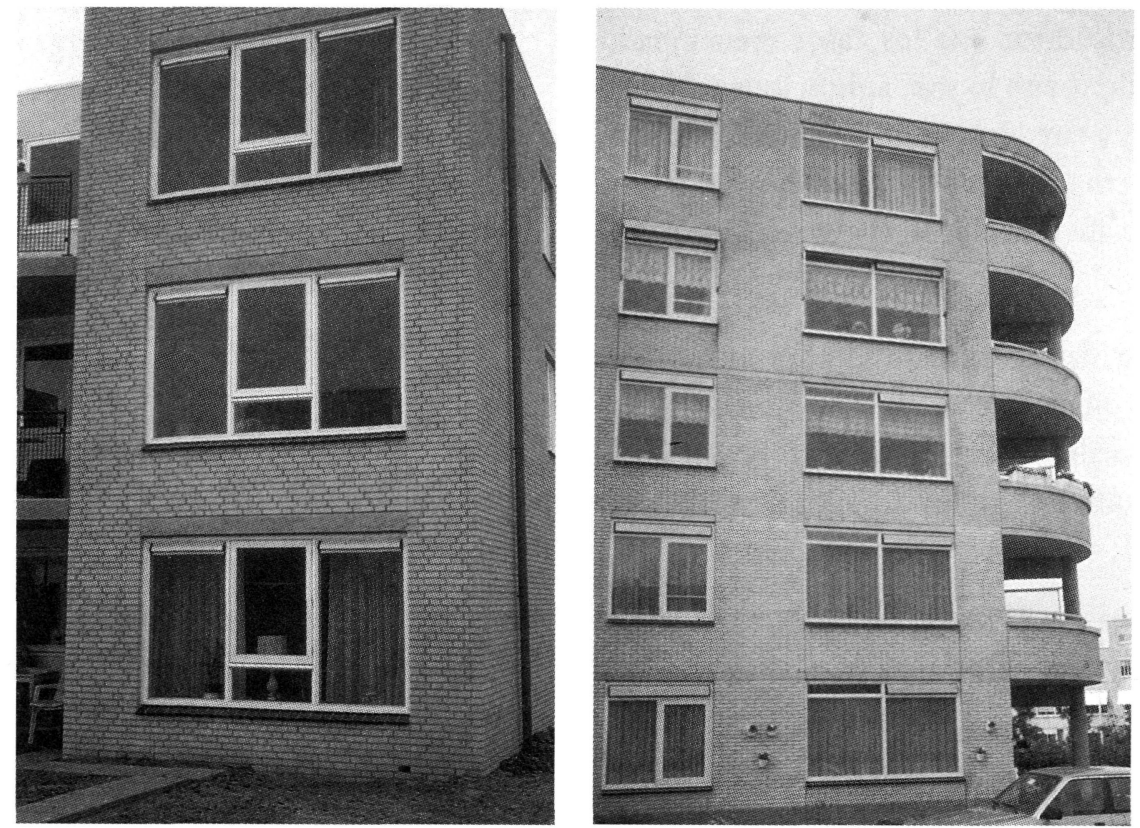

Fotos 2 y 3.-Típica preferencia de los Arquitectos holandeses de hacer juntas verticales a ambos lados de los huecos.

Otra ventaja es la limitación de tensiones debidas a variación de temperatura y retracción, especialmente si se aplican ladrillos sílico-calcáreos o de hormigón.

Sólo si no se desea la presencia de juntas verticales en estas esquinas es posible una aplicación correcta de Murfor.

El número de tendeles armados dependerá de los elementos conocidos como: luz de hueco, altura del dintel integrado y la carga aplicada.

A fin de evitar fisuras de una anchura inaceptablemente grande, se debe limitar la tensión del acero Murfor en el estado límite de capacidad de servicio, comparable con el diseño de estructuras de hormigón armado.

En la práctica se debe asumir una tensión de acero máxima de $200 \mathrm{~N} / \mathrm{mm}^{2}$ como valor medio para cálculos en el estado límite de capacidad de servicio.

La calidad de la albañilería y la clase de ladrillos influirá en la magnitud de esta tensión (Tabla 1).

También aparece, por los ensayos, que esta tensión es un valor medio aceptable para ladrillos hechos de arcilla, silicato de calcio u hormigón.

En opinión de los autores, una importante condición, para una posterior reducción de la mencionada tensión media del acero, será la posible aplicación de solapes que se han de aplicar en aberturas con una longitud de más de unos 2,50 m. La efectividad de tales solapes se

\section{TABLA 1}

ENSAYOS DE TENSIÓN EN FÁBRICAS DE LADRILLO (Zürich, 1984)

\begin{tabular}{|c|c|c|}
\hline \multirow{2}{*}{$\begin{array}{c}\text { TIPO DE PIEZA } \\
1 \times \mathrm{h} \times \mathrm{d}(\mathrm{mm})\end{array}$} & $\begin{array}{c}\text { RESULTADOS DE ENSAYOS } \\
\epsilon_{\mathrm{r}} \cdot 10^{3}\end{array}$ & $\begin{array}{c}\text { tensión del acero } \sigma_{\mathrm{S}} \\
\left(\mathrm{N} / \mathrm{mm}^{2}\right)\end{array}$ \\
\cline { 2 - 3 } & $0,10-0,15$ & $200-300$ \\
\hline $\begin{array}{c}\text { Ladrillo cerámico } \\
300 \times 190 \times 150\end{array}$ & $0,075-0,088$ & $150-175$ \\
\hline $\begin{array}{c}\text { Ladrillo sílico-calcáreo } \\
250 \times 180 \times 135\end{array}$ & \\
\hline
\end{tabular}

ENSAYOS A FLEXIÓN CON CARGA PUNTUAL (Bélgica, 1984)

\begin{tabular}{|c|c|c|c|c|}
\hline \multirow{2}{*}{$\begin{array}{c}\text { LUGAR } \\
\text { DEL } \\
\text { ENSAYO }\end{array}$} & $\begin{array}{c}\text { CLASE DE } \\
\text { LADRILLO } \\
1 \times \mathrm{h} \times \mathrm{d}\end{array}$ & LUZ & ALTURA & $\begin{array}{c}\text { TENSION DEL ACERO } \\
\text { EN LA 1. }{ }^{\mathrm{a}} \text { FISURA }\end{array}$ \\
\cline { 3 - 5 } & $\mathrm{mm}$ & $\mathrm{m}$ & $\mathrm{N} / \mathrm{mm}^{2}$ \\
\hline \multirow{2}{*}{$\begin{array}{c}\text { Lovaina } \\
1984\end{array}$} & $\begin{array}{c}\text { Sílico-calcáreo } \\
214 \times 102 \times 82\end{array}$ & 1,80 & 1,00 & 235 \\
\cline { 2 - 5 } & $\begin{array}{c}\text { Sílico-calcáreo } \\
327 \times 240 \times 147\end{array}$ & 3,00 & 0,74 & 160 \\
\hline \multirow{2}{*}{$\begin{array}{c}\text { Amberes } \\
1984\end{array}$} & $\begin{array}{c}\text { Sílico-calcáreo } \\
214 \times 102 \times 82\end{array}$ & 1,80 & 1,00 & 210 \\
\cline { 2 - 5 } & $\begin{array}{c}\text { Ślico-calcáreo } \\
327 \times 240 \times 147\end{array}$ & 3,00 & 0,74 & $160^{*}$ \\
\hline \multirow{2}{*}{$*$} \\
\hline
\end{tabular}



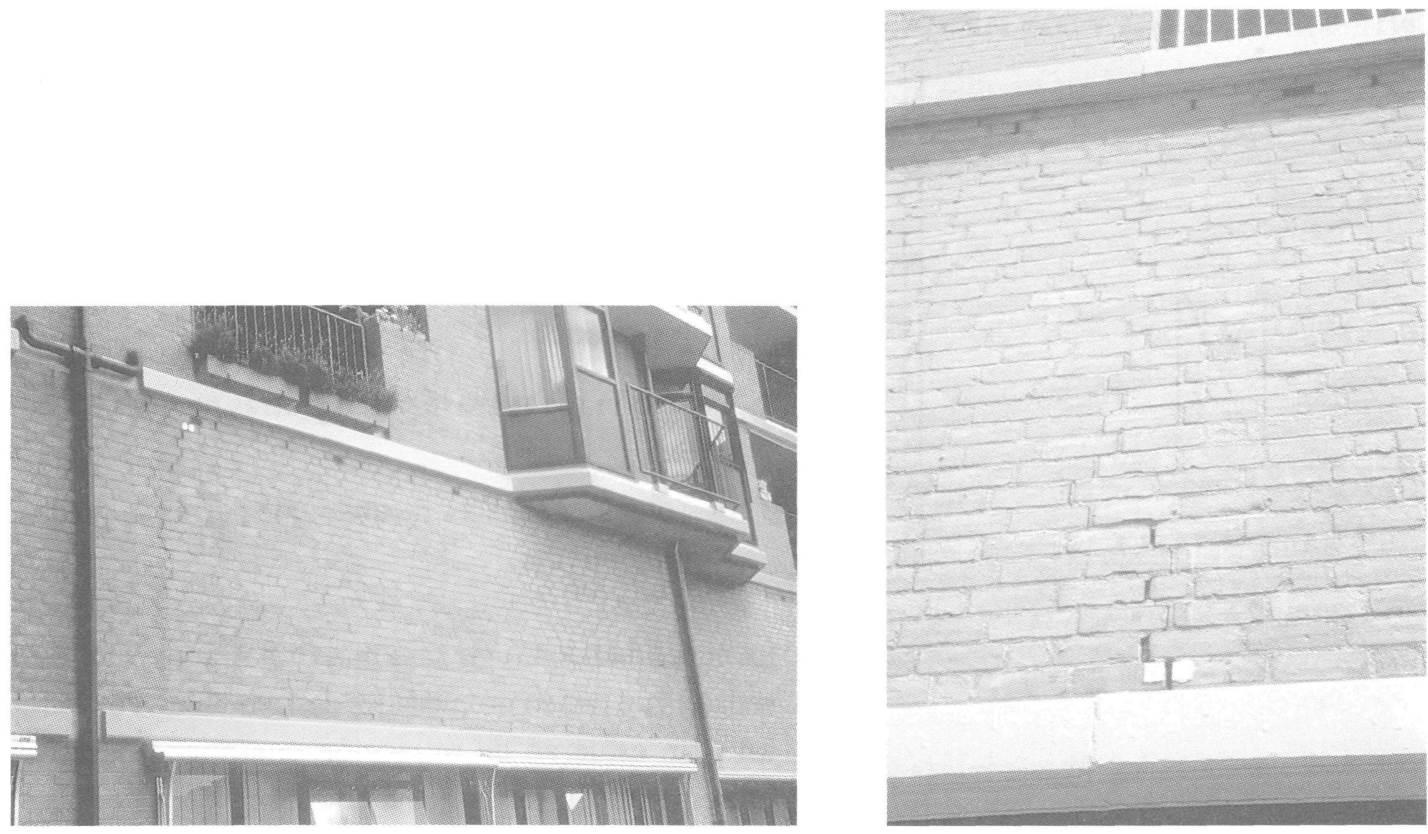

Fotos 4 y 5.-Fisuración de fábrica producida por ejecuciones incorrectas.

obtendría mediante una serie de ensayos, donde el material de fábrica y la longitud de solape de la armadura Murfor podrían ser parámetros importantes.

La limitación de anchura de fisura de los resultados de ensayo son también importantes para el diseño de particiones. La cantidad de tendeles armados en estas paredes se determina usualmente por comparación del comportamiento elástico de estos elementos, cargados por su propio peso en el plano. Teniendo en cuenta la longitud relativamente grande de estos tabiques, que corresponde a la longitud de la luz del forjado donde están apoyados, se requerirá al menos un solape por cada junta horizontal.

Si se ha de armar más de una junta horizontal los solapes deberán disponerse alternados y no se deben colocar en áreas de alta tensión.

A este respecto es importante mencionar que, en Holanda, se usan frecuentemente grandes bloques sílicocalcáreos y de hormigón en particiones. Otros dos problemas se pueden presentar si estos bloques tienen dimensiones corrientes de $500 \times 250 \mathrm{~mm}$ y una anchura entre 100 y $300 \mathrm{~mm}$ :

- La altura de $250 \mathrm{~mm}$ puede limitar la cantidad requerida de refuerzo en la región reservada para armado a tracción.
- El mortero de capa delgada, frecuentemente utilizado en los tendeles en vez de mortero de cemento, requiere la aplicación de la armadura Murfor de pletina. En la práctica holandesa el grueso de la pletina de $2 \mathrm{~mm}$ parece ser excesivo para el espesor aplicado normalmente en los tendeles, y esto podría ser la causa de fisuras horizontales en ellos.

Recientemente BEKAERT desarrolló la armadura de pletina con 1,5 $\mathrm{mm}$ de grueso, que debe impedir el posible apoyo de los bloques sobre la armadura.

La presencia de posibles restricciones de movimiento, debidas a ejecuciones incorrectas, es un tema importante para el desarrollo de fisuras en estas paredes y, en general, para detallar estructuras de fábrica. La aplicación de Murfor en tales paredes es una obligación. Si no se utiliza, la consecuencia es la formación de fisuras con anchuras inaceptables. A veces, cargas verticales impensables desde niveles más altos y efectos de temperatura son razones que pueden inducir a la fisuración (Fotos 4 y 5$)$.

Las paredes colocadas en la dirección del forjado pueden originar restricciones debido a:

- Adhesión a suelos de hormigón sobre las que están colocados:

- Solución: aplicación de cinta no adhesiva por debajo de la longitud total del tabique. 
TABIQUE INTERIOR SIN CARGA Y CON PUERTA

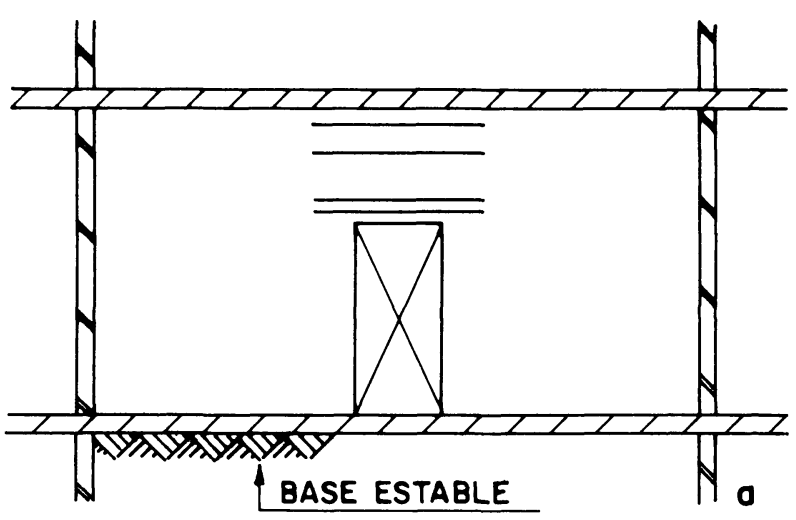

REFUERZO LOCALIZADO

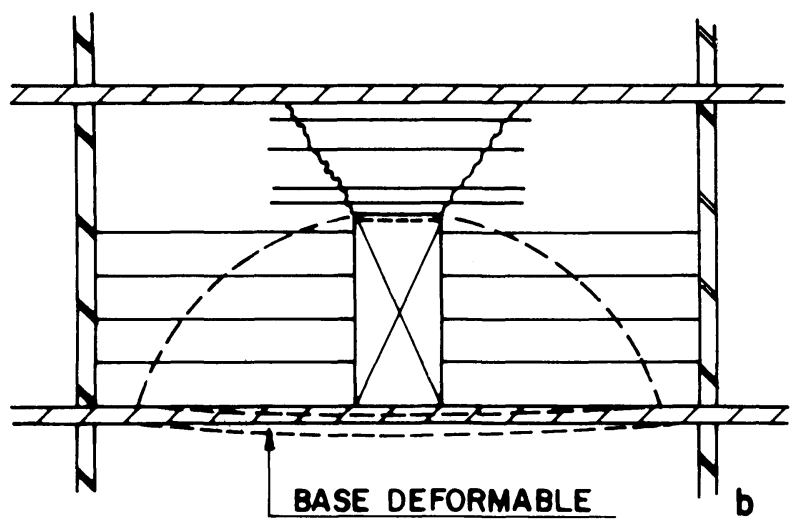

ARMADO HOMOGENEO + REFUERZO CONTRA FISURACIÓN

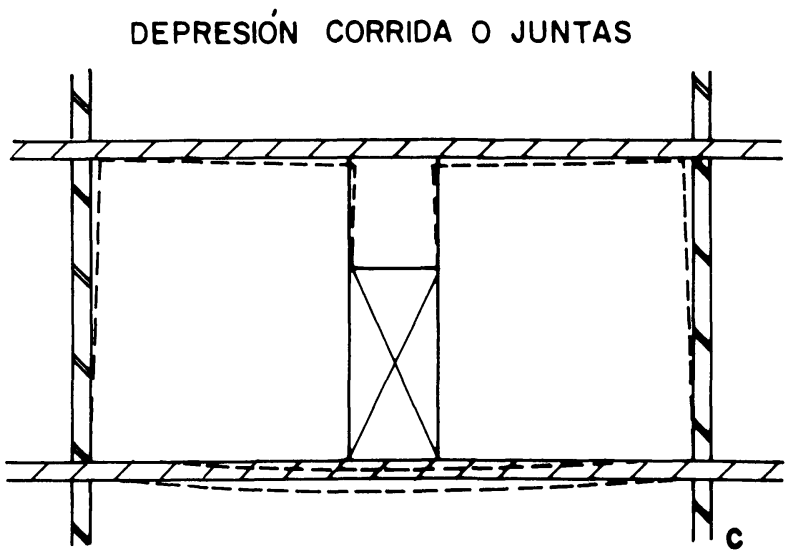

SOLUCIÓN ARQUITECTÓNICA ALTERNATIMA CON POSIBILIDAD DE DEFORMACIONES

Fig. 2.-Forma de evitar la fisuración en tabiquería.
- Carga vertical en la parte superior de las paredes internas, debido a la flecha no prevista de la estructura. Por ejemplo por deformación inelástica de suelos de hormigón:

- Solución: aplicación de junta en el tendel del hormigón con material aislante compresible y anclajes elásticos.

- Conexión fija entre la pared y otras paredes transversales:

- Solución: anclajes flexibles que permitan grados de movimiento.

La necesidad de armar será más importante para los materiales que tienen propiedades desfavorables. Por ejemplo un gran esfuerzo de retracción que puede llevar a un incremento de tensiones a tracción.

Las aberturas en tabiques, por ejemplo debido a puertas, deben ser tratadas con cuidado aplicando las armaduras con detalles arquitectónicos adecuados (Fig. 2).

Aunque el refuerzo se aplica localmente, o uniformemente repartido sobre la longitud total de esta pared interna, será razonable advertir la aparición de fisuras relativamente grandes, especialmente si este tabique está situado sobre un suelo o una viga que fleche. En ambos lados de la apertura de la puerta, partes del tabique están de hecho colgando de las paredes restantes (líneas de trazos) lo cual en la práctica holandesa no puede ser atado con armado vertical.

En la figura 2, se ha dado una solución alternativa que permite deformaciones en el plano de la pared.

Pienso que el consejo del consultor debe ser honesto, avisando al cliente de que, a pesar del refuerzo aplicado, la formación de fisuras podría producirse; pero que sin las armaduras, las grietas serán ciertamente más largas y con mayor anchura y, por tanto, más visibles.

La elección del tipo de pieza puede influir también en la anchura de las fisuras, especialmente si la altura total de una sección vertical es bastante pequeña. Una altura menor de ladrillo será una elección mejor en tal caso (Figs. 3 y 4).

El modo en que los problemas se presentan al consultor de la fábrica armada Murfor es, a menudo, muy incompleto y poco claro. 

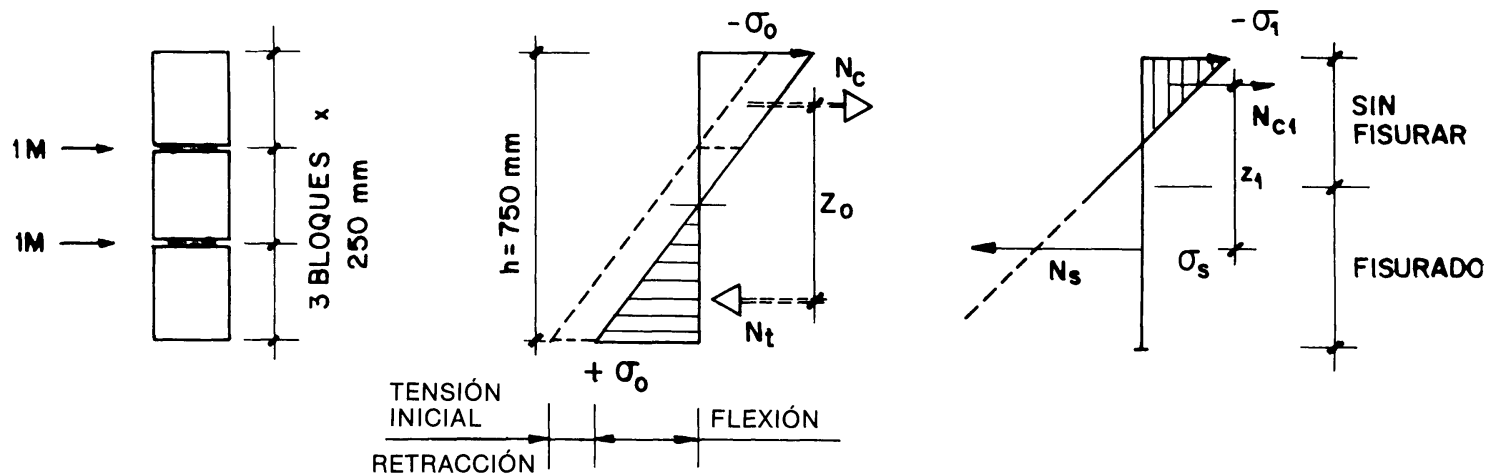

Fig. 3.-Distribución desfavorable del armado con respecto a la limitación de la anchura de fisuración debida a la gran dimensión de los bloques (separación entre tendeles).

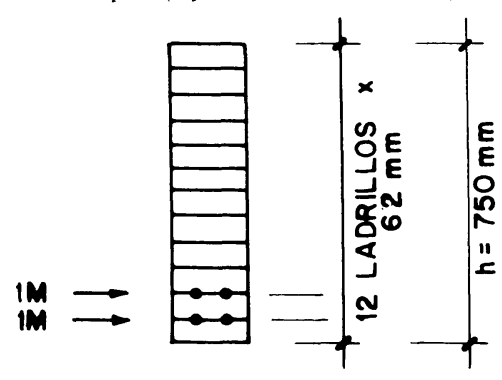

SECCIÓN VERTICAL DEL DINTEL

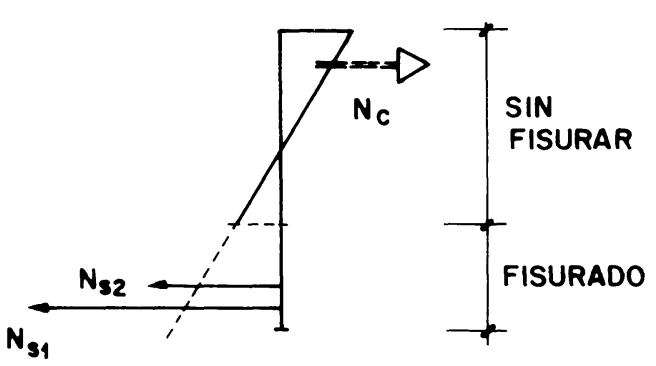

DIAGRAMA DE TENSIÓN JUSTO DESPUÉS DE LA 1. FISURA

Fig. 4.-Distribución favorables del armado con respecto al límite de la anchura de fisuración si se pueden escoger ladrillos pequeños.

Como un ejemplo confuso, véase cómo circunstancias adicionales pueden influir en la distribución de momentos, tensión de corte y tensión normal.

La información por teléfono es a menudo pobre y se describe como "algunas aberturas pequeñas aisladas". Llamemos a ello el problema aislado que se ve en la figura 5:

- una abertura de 1,5 m aproximadamente,

- altura del dintel $\mathrm{h}=0,9 \mathrm{~m}$ y

- anchura de la pared doble externa (100 mm).

Como consecuencia de una consulta, resultó ser, al parecer, parte de una pared frontal más grande con muchas otras ventanas, y una gran abertura en el primer piso, a la que llamamos el problema integrado.

Será evidente que la consulta tendrá diferentes resultados. Así, la falta de información por parte del cliente puede tener consecuencias desfavorables para la colocación de las armaduras y la formación de fisuras en la realidad.

En cuanto al armado de muros cargados horizontalmente, sólo se pueden utilizar para sótanos en las regiones del Este y del Sur de Holanda. La razón es que el nivel del agua subterránea en otras regiones es demasiado elevado para estar seguros de tener un sótano seco.

Una dificultad en el diseño de muros de sótano es que la aplicación de un refuerzo ortogonal no es fácil de realizar. Normalmente la distribución elástica de momentos en estos muros, con luces en dos sentidos, debe ser pasada por alto si sólo puede proporcionar armado Murfor horizontal. Esto puede llevar a fisuras de anchura indeseada en el estado límite de servicio (Fig. 6). 


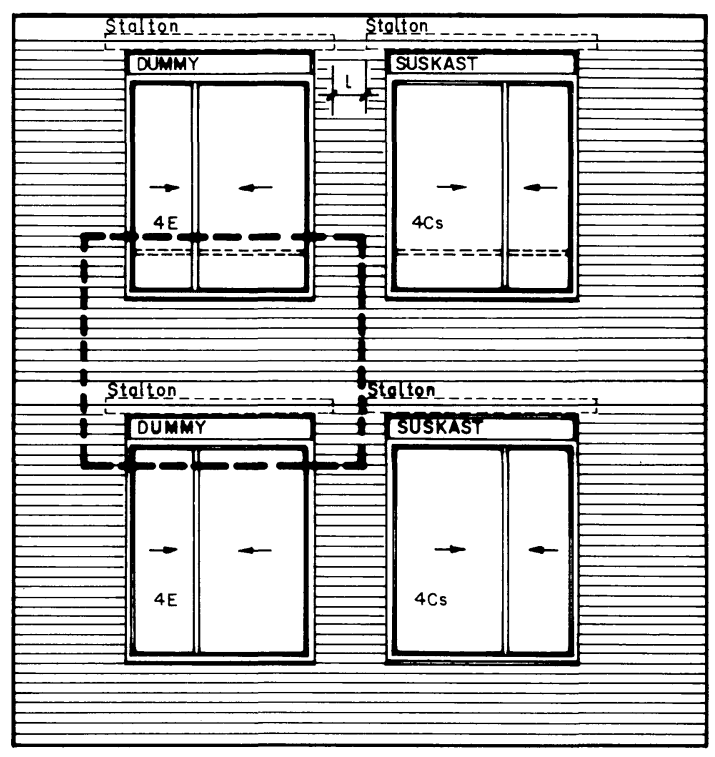

Fig. 5.-Dintel típico de fábrica armada.

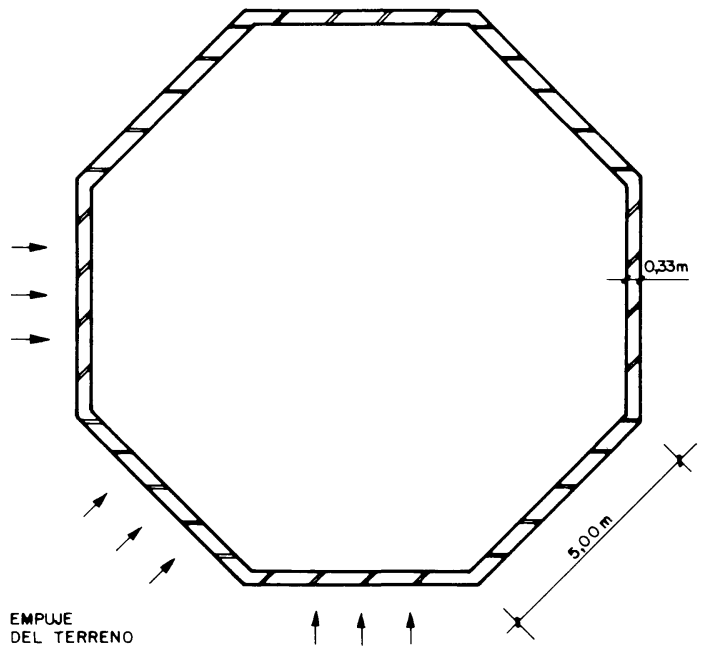

Fig. 6.-Sótano octogonal para absorber eficazmente los empujes del terreno, armando los tendeles.

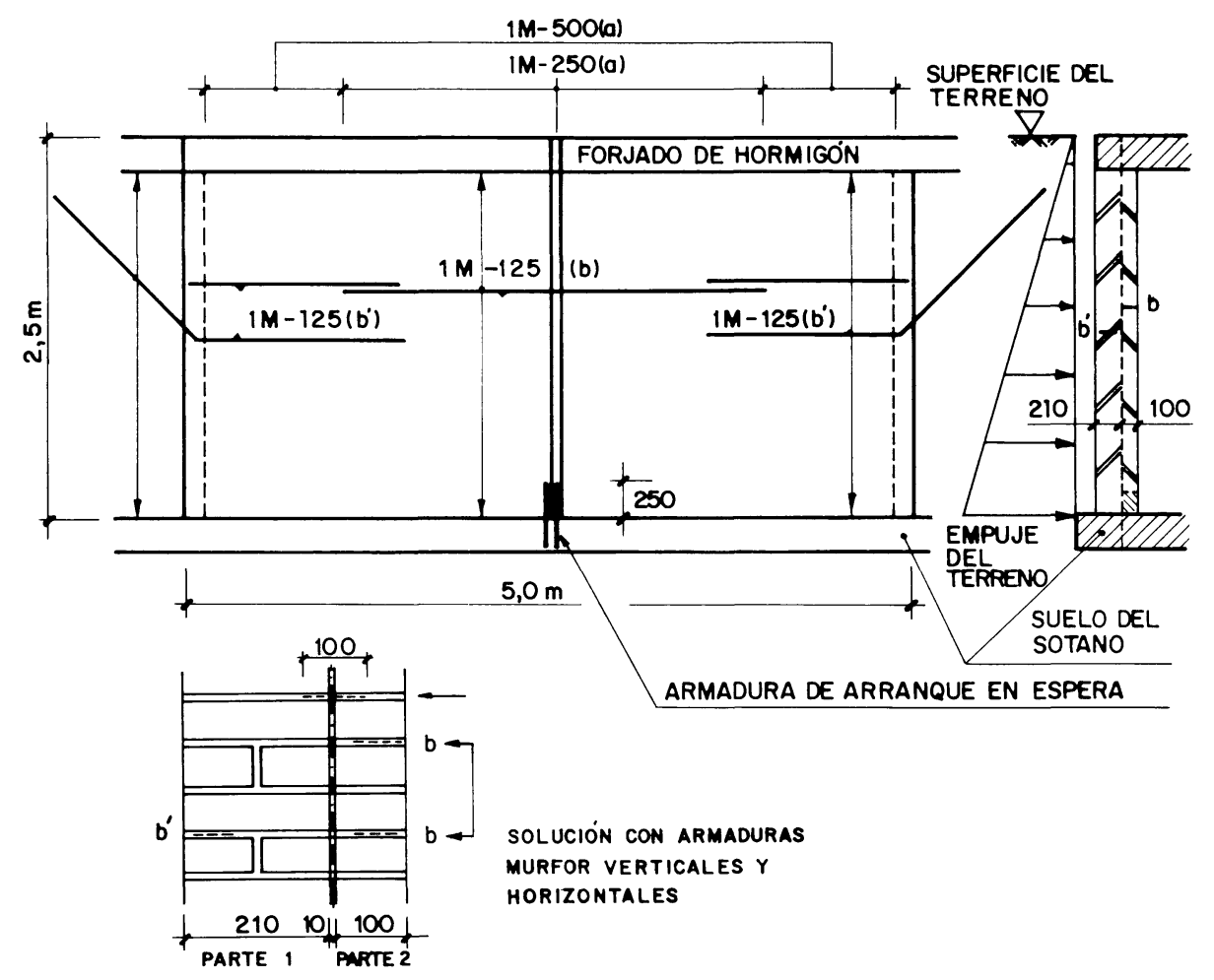

Fig. 7.-Solución de muro doblado para sótanos con armaduras Murfor colocadas horizontal y verticalmente.

Se puede ofrecer una alternativa mejor usando un muro doblado de $320 \mathrm{~mm}$ de grueso con ladrillos normales $(215 \times 103 \times 52 \mathrm{~mm})$. Se construye primero la hoja del muro con una anchura de $210 \mathrm{~mm}$. Después se coloca la segunda de $110 \mathrm{~mm}$, que se acopla con armaduras Murfor de $100 \mathrm{~mm}$ colocadas previamente en los tendeles de la hoja de $210 \mathrm{~mm}$. Las armaduras Murfor verticales requeridas se colocan en la junta vertical co- mún entre ambas hojas de fábrica impidiendo que se formen excesivas fisuras horizontales (Fig. 7).

Se ha intentado ofrecer una perspectiva de cómo se pueden impedir las fisuras en Holanda, o cómo anticiparse a la probable aparición de fisuras en estructuras de obra de fábrica. El control de la fisuración con armaduras Murfor, en los casos donde las fisuras puedan ser inevitables, es pues una solución razonable. 